\title{
Editorial: Five Years of Physical Review Fluids
}

Physical Review Fluids (PRFluids) launched on May 6, 2016, under the editorial leadership of John Kim and Gary Leal, publishing five research papers and a short Editorial expressing the support of the APS DFD for the new journal [1]. In celebration of this fifth anniversary, we take the opportunity to reflect on the journal's mission, achievements, and evolution.

\section{PRFluids is growing and evolving}

The journal has grown steadily over five years, thanks to the community's support. Today's monthly issue is roughly four times the size of our first issue, with a healthy mixture of Articles and Letters-a short format reserved for particularly important results and fast-track publication. Since its inception, PRFluids has published papers from authors in 65 countries and on a variety of topics, as illustrated in Fig. 1; the full list of topics covered by the journal is available online [2].

\section{PRFluids is publishing pioneering and high-impact studies in fluid mechanics}

PRFluids is the home for fundamental research in fluid mechanics. We strive to promote modern and original approaches to not only long-established research but also nontraditional and emerging areas, with a wide scope of applications. We offer unique breadth in the subject, from fundamental investigations to multidisciplinary studies bridging the field with other areas of science, where insights from fluid dynamics arise in a vast array of problems, for example, in health, energy, climate, the COVID-19 pandemic, computer science, and soft matter and biophysics, to name just a few.

The editorial team has worked together to showcase research in rapidly growing areas. One example is the use of artificial intelligence (AI) to understand large datasets in fluid mechanics research. Data-driven analysis and machine-learning tools have, for example, provided dramatic improvement in modeling the Reynolds stresses using Reynolds-averaged Navier-Stokes (RANS) approaches as well as exciting potential for the prediction of temporally evolving turbulent shear flows using, for example, neural networks. In an Editorial [3], Associate Editors Michael Brenner and Petros Koumoutsakos have outlined the PRFluids criteria for successful manuscripts in this area, which use AI to gain physical insight and interpretability. An earlier Perspective article [4] elaborated exciting areas for potential advances as well as some of the outstanding challenges.

In the active and broad area of turbulence, the journal has published work on pushing the limits of high-resolution simulations and creating invaluable datasets 


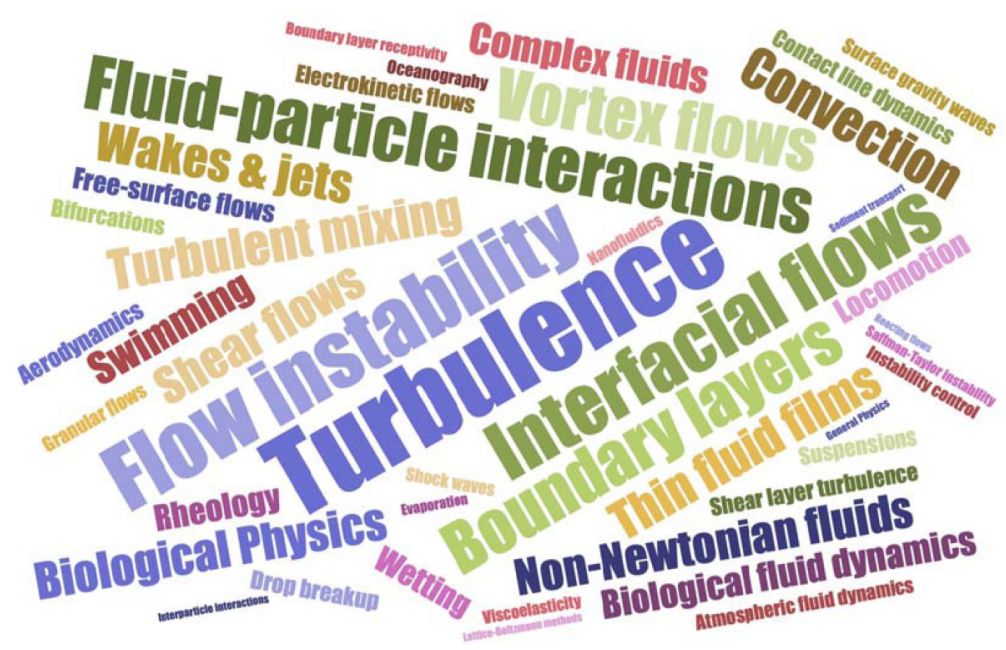

FIG. 1. This "word cloud" shows the distribution of topics appearing in PRFluids papers. The topics are taken from the PhySH taxonomy scheme used to classify papers in APS journals.

in classical turbulence while also providing new experimental observations, estimation techniques, and physically insightful simplified models of quantities of engineering importance. In multiphase flows, direct numerical simulations have been used to identify closures for bubble-induced turbulence in disperse bubbly flows and have uncovered mechanisms for instability in rising bubbles. In the area of high-speed flow, nonequilibrium direct molecular simulation has been employed for ab initio molecular-level investigations of the $\mathrm{N}_{2}+\mathrm{N}_{2}$ and $\mathrm{N}+\mathrm{N}_{2}$ processes of nitrogen dissociation that can take place concurrently. A recent Perspective article [5] and related Journal Club [6] event elaborate upon current advances in geophysical and environmental fluid mechanics.

On the other side of the spectrum in Reynolds numbers, a lot of novel work has appeared in capillary flows, from a new method to remove nanodroplets from superhydrophobic surfaces to novel insight on the age-old problem of bubble bursting. New studies have also demonstrated how surfaces impregnated with another liquid can enhance thermocapillary motion and how to quantify their flow-driven failure. Papers published in PRFluids on the fluid mechanics of porous media have confirmed a 20-year-old prediction on the impact of wetting on the instability between two fluids in a porous medium, proposed a new geometric state equation for the capillary pressure for two-fluid flow in porous media, and elaborated on a new way to relate the structure of individual pores to macroscopic flow. Studies appearing in the journal have also advanced our understanding of nonNewtonian fluid mechanics, with new discoveries in viscoelastic capillary flows, suspensions, and elastic turbulence. The journal has also seen a lot of activity in the field of biological fluid mechanics, with the discovery of new instabilities in the flow of bacteria, the synchronization of cellular flagella, the biomimetic propulsion of magnetic particles, and illumination of the fluid dynamics near fish skin. 


\section{PRFluids is a journal with global reach and a special relationship with the DFD of APS}

We are part of the Physical Review family and are published by APS, a nonprofit professional society committed to scientific independence. Growing out of the DFD in 2016, we are now established as a leading and global journal run by the community for the community. The connection with the DFD remains important to the journal, and it is substantial; the editorial team includes former DFD Chairs as well as past winners of the Fluid Dynamics Prize [7], the Stanley Corrsin Award [8], the Frenkiel Award (for papers published in the journal by early career researchers [9]), and the Andreas Acrivos Dissertation Award [10]. The journal also enjoys the support of a distinguished and diverse editorial board ranging from early-career scientists to senior members of the community. The journal and its editorial team remain active in the DFD on many fronts. By publishing timely invited DFD papers and Gallery of Fluid Motion submissions [11], we ensure that we are always at the forefront of trends in the community. The journal provides leadership to the community on emerging topics in fluid dynamics, for instance through our sponsorship of Fluids Next sessions at the annual DFD meeting and associated special paper collections. As an example, our collection on the Fluid Dynamics of Infectious Diseases, gathered after a Fluids Next event held at the 2020 DFD meeting, sheds important light on fluid mechanics phenomena relevant to the COVID-19 pandemic [12].

\section{PRFluids is a journal with a mandate to provide leadership in the fluid dynamics community and reflects the evolution and progress of research in the field}

Researchers will find many reasons to publish their research in PRFluids. First, the journal provides leadership in the broader fluid dynamics community by fostering ideas that stimulate and enrich the field and by publishing papers that discover, explain, and help us understand fundamental physical phenomena (see Ref. [2] for our mission). In order to seed, cultivate and define new areas of investigation, we are continuously evolving our stance on what constitutes a PRFluids paper. For example, some scientific problems may require greater latitude for innovative or even risky approaches. However, PRFluids maintains high standards with regard to the scientific advance required for publication, and authors must contribute to "why" and "how," not just "what," in their descriptions of physical phenomena.

Second, manuscripts submitted to PRFluids are handled by technical leaders in the field; the journal offers fast publication and transparent peer review [13]. Our knowledgeable, international team of editors ensures that innovative and rigorous science is published and highlighted in a timely manner and that the journal is open to all kinds of expression. The team also strives to engage the community in the reviewing and quality control process. Editors publish regularly in the journal themselves. 
Finally, PRFluids papers reach beyond the traditional fluid dynamics community. As a part of the Physical Review family, PRFluids has a broad and global readership across physics, engineering, and applied mathematics, which gives our papers high visibility to a wide audience. PRFluids is also actively promoted in the broader scientific community. Selected papers are highlighted in every issue as Editors' Suggestions, and PRFluids papers are regularly highlighted with stories in the free online APS magazine Physics or covered in broader media outlets. The Gallery of Fluid Motion showcases the elegant visual elements of our field to engage students and the public alike. PRFluids also participates actively in the APS Journal Club [6], where select papers are highlighted through live presentations, with broad viewing via YouTube recordings; recent topics have ranged from icebergs and effervescence to respiratory droplets and turbulence.

We look forward to the next five years and beyond of scientific discovery and innovation in PRFluids.

Eric Lauga and Beverley McKeon, Co-Lead Editors

Brad Rubin, Journal Manager

Guido Boffetta, Michael Brenner, Cecile Cottin-Bizonne, Luminita Danaila, Nicolas Hadjiconstantinou, Guowei He,

Petros Koumoutsakos, Sanjiva Lele, Eckart Meiburg, David Quere, Peter Schmid, Eric Shaqfeh, Jacco Snoeijer, Howard Stone, Bruce Sutherland, Emmanuel Villermaux, Roberto Zenit, Associate Editors

Published 1 December 2021

DOI: 10.1103/PhysRevFluids.6.120001

[1] https://journals.aps.org/prfluids/

[2] https://journals.aps.org/prfluids/about

[3] https://journals.aps.org/prfluids/edannounce/10.1103/PhysRevFluids.6.070001

[4] https://journals.aps.org/prfluids/abstract/10.1103/PhysRevFluids.4.100501

[5] https://journals.aps.org/prfluids/abstract/10.1103/PhysRevFluids.6.020501

[6] https://journals.aps.org/journal-club

[7] https://www.aps.org/programs/honors/prizes/fluid.cfm

[8] https://www.aps.org/programs/honors/prizes/corrsin.cfm

[9] https://engage.aps.org/dfd/honors/prizes-awards/frenkiel-award

[10] https://www.aps.org/programs/honors/prizes/acrivos.cfm

[11] https://gfm.aps.org

[12] https://journals.aps.org/prfluids/collections/fluid-dynamics-of-infectious-diseases

[13] https://journals.aps.org/prfluids/authors/editorial-policies-practices 